\title{
On The Chaotic Nature of Solar Activity
}

\author{
J. Kurths \\ Institute of Astrophysics, D-0-1501, Tremsdorf, Germany \\ U. Feudel and W. Jansen \\ Institute of Cybe Rnetics and Information-Processes, \\ D-0-1086, Berlin, Germany
}

\section{ABSTRACT}

Applying modern techniques of time series analysis, there are serious indications that the dynamics of the global solar activity is a low dimensional chaos. A simple non-1inear dynamo model is qualitatively studied exhibiting a rich dymamical behaviour from steady state via some bifurcation to a chaotic regime.

\section{INTRODUCT ION}

The energy output of the sun as the main basis of 1 ife on the earth is nearly constant. However, the visible solar surface, the photosphere, is far from being uniform. It always consists of a granulation pattern which is produced by buo yancy-driven convection. Furthermore, there occur sunspots located within active regions of the photosphere. These are dark regions, in which the luminosity is diminished with respect to the general solar surface. In these regions strong magnetic fields (1-10 kilogauss) appear, whereas the global magnetic field of the sun is of the order 1 gauss, only. As was discovered by the druggist $\mathrm{H} . \mathrm{Sch}$ wa be in 1843, the spatial distribution of sunspots reflects in average a 11-year cycle of solar activity. To quantify the dynamics of the solar activity $R$. Wolf introduced the sunspot relative numbers Which can be regarded as a rough measure of the evolution of the solar magnetic field (Priest, 1982).

Note that the dynamics of $W$ is far from being periodic(Fig.1) We observe a complicate amplitude-frequency modulation as 


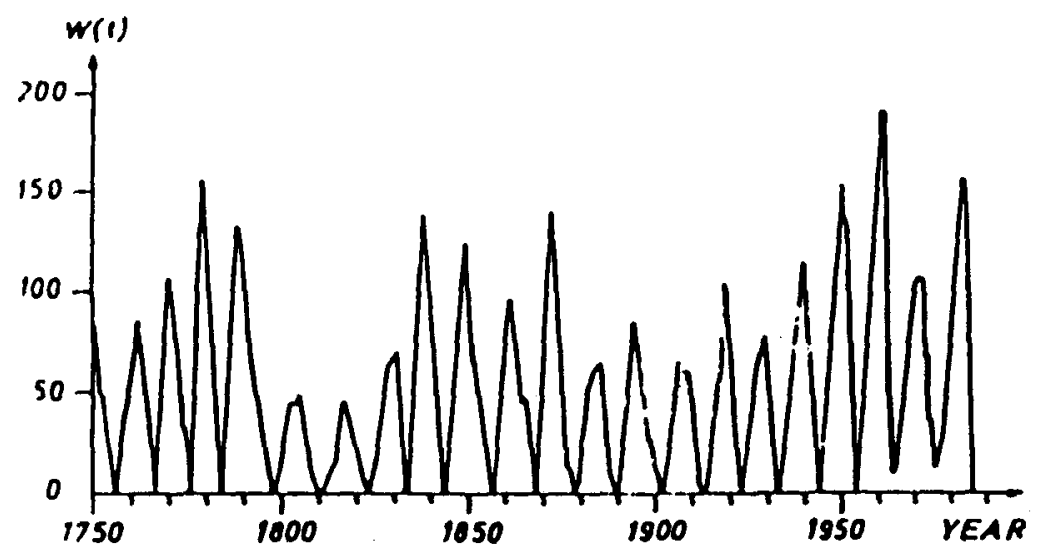

Fig. 1: Yearly sunspot relative numbers W(t) (Wolf numbers)

well as intervals of extremely low solar activity, such as the Maunder minimum during 1645-1715 (Eddy, 1976). To explain these peculiar features of the solar cycle a nonlinear theory is necessary.

The purpose of this contribution is to $f$ ind out nonl inear interactions generating such dynamics. First, we apply methods of time series analysis recently developed in the theory of nonlinear systems to estimate dynamical invariants from the Wolf numbers. Chapter 3 contains the qualitative analysis of a nonlinear dynamo model. Finally, in Chapter 4 we compare these findings in order to judge the suitability of the model.

\section{ANALYSIS OF THE SUNSPOT NUMBERS}

Dynamical systems theory has provided techniques to analyze seemingly irregular time series. By calculating their dynamical invariants, such as attractor dimensions or Lyapunov exponents, they allow to decide whether the under1ying system is low-dimensional and/or chaotic (Ruelle,1989).

In order to extract these quantities from the scalar Wolf numbers $W$, a reconstruction of the phase space using the embedding method by Takens (1980) is necessary. Grassberger and Procaccia (1983) proposed a simple technique to search for Hausdorff-like dimensions, the correlation dimensions. Applying this procedure to the Wolf numbers we $f$ ind in fact a low correlation dimension $\bar{D}_{2}=2.1 \pm 0.3$ (Kurths, 1987 ).

A study on for ecasting the sunspot numbers by means of learning nonlinear dynamics, as proposed by Farmer and 
Sidorovich (1987), gives further evidence for the chaotic nature of solar activity (Kurths and Ruzmaikin,1990). We have shown that this rather simple approach yields relatively good results for short-term forecasts ( $<11 \mathrm{yr})$. Note that this procedure is superior to linear prediction models. On the other hand, the average prediction error grows considerably for longer forecasts (Fig. 2). This is a typical property of chaotic systems which preclude long-term predictions.

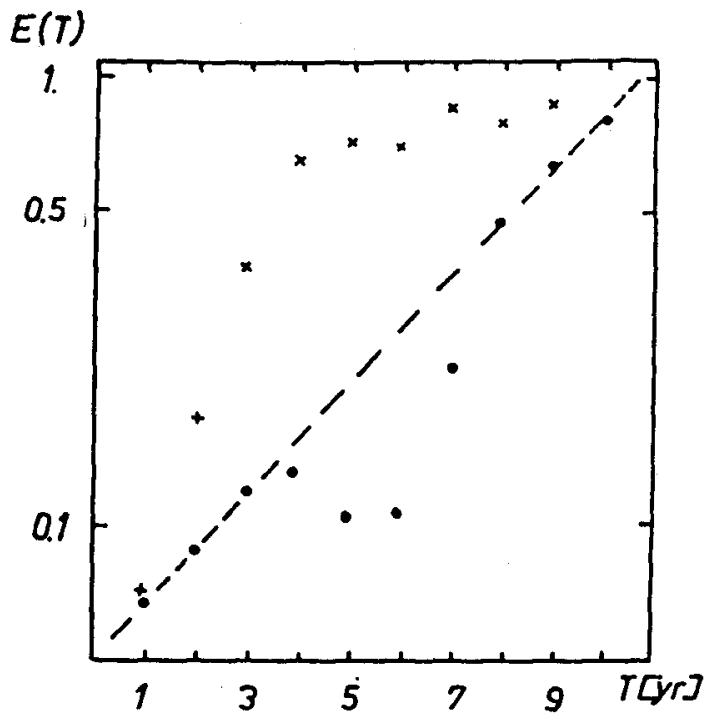

Fig. 2: Average prediction error $E(t)$ of the yearly Wolf numbers as obtained from fitting o a model learning nonlinear dynamics, $x$ a global 1 inear model, $T$ is the forecasting time.

These findings suggest that the dynamics of the global solar activity is low-dimensional chaos.

\section{A SIMPL IFIED NON INEAR DYNAMO MODEL FOR SOLAR ACTIVITY}

Large-scale variations of the solar activity can be explained in terms of a mean-field dynamo theory. The basic idea of solar dynamo theory is that the magnetic field of the sun is amplified and maintained by its rotation (Krause and Radler, 1980). The rotation of a shell, the solar convection zone, with the angular velocity $\Omega$ in the magnetic field 
B leads to an electric field in radial direction. Due to this field the charged particles move outward and in the conducting wire a current begins to flow. This current causes a new magnetic field by itself whose direction is equal to that of the original magnetic field $B$.

The only physical mechanism known to produce such magnetic variations are hydrodynamic plasma motions, that means differential rotation and turbulent convection. The changes of the field $B$ are described by the induction equation. For convenience, the mean magnetic field is split into two parts, the poloidal component $B_{p}$ and the toroidal component $B_{t}$

$$
\vec{B}=\vec{B}_{t}+\Delta \times \vec{A}_{t}
$$

The poloidal component is expressed as the rotation of a purely toroidal vector potential $A_{t}$. This way we get equations for the dynamics of $A_{t}$ and $B_{t}$

$$
\begin{aligned}
& \frac{\partial \vec{A}_{t}}{\partial t}=(\alpha+C) \vec{B}_{t}+\Delta \vec{A}_{t} \\
& \frac{\partial \vec{B}_{t}}{\partial t}=D \nabla A_{t}+\Delta \vec{B}_{t}
\end{aligned}
$$

including the influence of the mean helicity $d$. The dynamo number $D$ depends directly on the differential rotation and $t$ he mean helicity and is inversely proportional to the square of the coefficient of turbulent diffusivity. Additionally, the back-action of the magnetic field upon the helicity is included. Eq. (2b) describes the changes of the helicity

$$
\frac{\partial C}{\partial t}=-V C+p \vec{A}_{t} \vec{B}_{t}-q(\alpha+C) \vec{E}_{t}^{2}
$$

The parameters $V, p, q$ control the relaxation of $c$ resp. the influence of the nonlinear terms. C is the deviation of the helicity from its value in the absence of the magnetic field. Thus we get a nonl inear dynamo model. To make it tractable the equations are truncated using a first order mode ansatz yielding a system of 7 autonomus differential equations depending on seven coefficients (Malinetsky et al., 1986)

$$
\begin{aligned}
& \dot{a}_{1}=-\sigma a_{1}+\left(\alpha+c_{0}\right) b_{1}+0.5\left(c_{1} b_{1}+c_{2} b_{2}\right) \\
& \dot{a}_{2}=-\sigma a_{2}+\left(\alpha+c_{0}\right) b_{2}-0.5\left(c_{1} b_{1}-c_{2} b_{2}\right) \\
& \dot{b}_{1}=-b_{1}-D a_{2}
\end{aligned}
$$




$$
\begin{aligned}
& \dot{b}_{2}=-b_{2}+D a_{1} \\
& \dot{c}_{0}=-v_{0} c_{0}+p\left(a_{1} b_{1}+a_{2} b_{2}\right)-q\left(\left(\alpha+c_{0}\right)\left(b_{1}^{2}+b_{2}^{2}\right)\right. \\
&\left.+0.5 c_{1}\left(b_{1}^{2}-b_{2}^{2}\right)+c_{2} b_{1} b_{2}\right) \\
& \dot{c}_{1}=-V c_{1}+p\left(a_{1} b_{1}-a_{2} b_{2}\right)-q\left(\left(\alpha+c_{0}\right)\left(b_{1}^{2}-b_{2}^{2}\right)\right. \\
&\left.+c_{1}\left(b_{1}^{2}+b_{2}^{2}\right)\right) \\
& \dot{c}_{2}=-V c_{2}+p\left(a_{1} b_{2}+a_{2} b_{1}\right)-q\left(\left(\alpha+c_{0}\right) 2 b_{1} b_{2}+c_{2}\left(b_{1}^{2}+b_{2}^{2}\right)\right)
\end{aligned}
$$

\section{QUAL ITAT IVE ANAL YSIS OF THE NON INEAR DYNAMO MODEL}

In order to investigate the qualitative behaviour of the dynamo model (3) in dependence on characteristic parameters this 7-dimensional system of ordinary differential equations has been analyzed by using the software system CANDYS/QA designed for the qualitative analysis of nonl inear dynamical systems (Feudel and Jansen,1988). This leads to the construction of a bifurcation diagram.

For the model ( 3 ) the dynamo number $D$ is the crucial bifurcation parameter. Moreover, the special values $D$ at which qualitative changes occur mainly depend on the parameter $q$. Hence, our results are shown in a corresponding D-q-bifurcation diagram (Fig.3). It exhibits 6 regions with different kinds of invariant sets. It is obvious that this system possesses a trivial stationary point where all variables are equal to a vanishing magnetic field. At a $D_{0}$ the system undergoes a lopf bifurcation: the trivial stationary point looses its stability and a stable periodic solution occurs. This stable cycle has been continued with increasing dyamo number. At a critical value $D_{1}$ this periodic solution looses its stability too and we find a stable quasiperiodic motion on a torus which passes to a stable three frequency torus for higher $D$. Such stable three frequency tor 1 have been reported till now for a few systems, only (Kaneko, 1986, Battel ino, 1988, Nicolis, 1990). As is kno wn from special maps (Kaneko, 1986) this torus merges in a two frequency torus if $D$ increases further. For higher $D$ a new instability sets in leading to a chaotic region.

Since the attractor is somewhat complicated, we cannot decide from Poincare plots whether the motion is quasiperiodic or chaotic. A conclusive way to distinguish between torus and chaos is to estimate the Lyapunov exponents $\lambda_{i}$. They express this by the exponential convergence or divergence of initially nearby trajectories, i.e. for the distance of two trajectories holds after an evolution time $T$ 


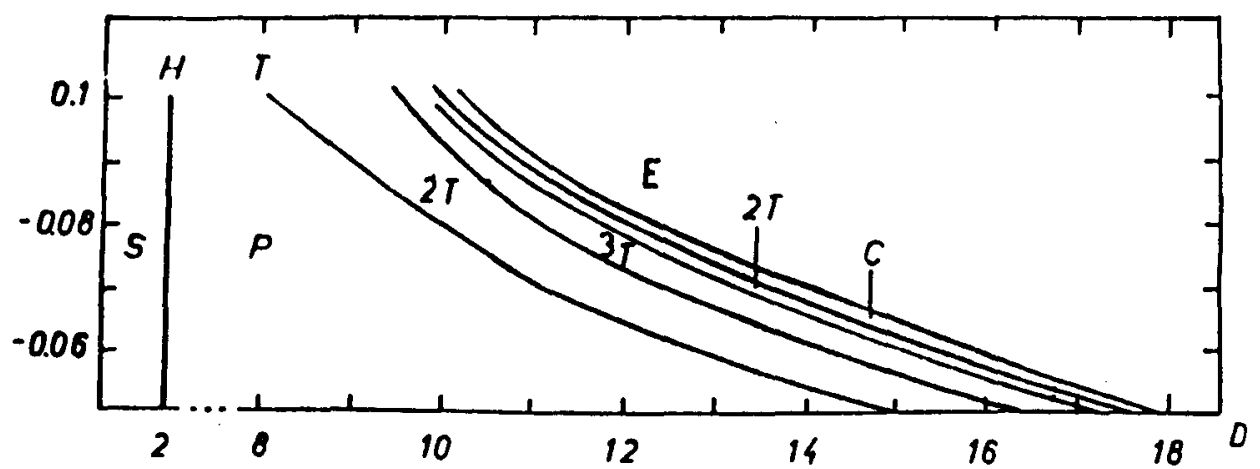

Fig. 3: Bifurcation diagram for the nonlinear dynamo model (3) in dependence on the dynamo number $D$ and parameter q, S - stationary solution, $p$ - periodic solution H - Hopf bifurcation, 2T - stable two-torus, 3T stable three-torus, C - chaos, E - exploding.

$$
d(T) \sim e^{\lambda T}
$$

where $\lambda<0$ means stability $\lambda>0$ points to unstable behaviour. If the maximum Lyapunov exponent $\lambda_{\max }$ is positive this stage of the system is defined to be chaotic.

The $\lambda$, of the model (3) are estimated applying the numerical procedure proposed by Shimada and Nagashima (1979).

Between the $\lambda$ of a trajectory of a system and the dimension of the corresponding attractor exists a relation found by Kaplan and Yorke (1979). If the $\lambda_{i}$ are ordered descendingly,

$$
\mathrm{D}_{\mathrm{KY}}=\mathbf{k}-\sum_{\mathbf{i}=1}^{\mathrm{k}} \lambda_{i} / \lambda_{\mathbf{k}+1}
$$

provides a good approximation of the Hausdorff dimension. $k$ is the greatest number with $\sum_{i=1}^{k} \lambda_{i}>0$. 
Calculating the $\lambda_{i}$ we indeed $f$ ind a region for $D$ and $q$ with chaotic motion. In the case of stable tor $i D_{K Y}$ is equal the number of incommensurate frequencies, but it takes fractal values in the chaotic regime (Fig. 4). The transition from quasiperiodicity to chaos found for the system (5) is the typical Newhouse-Ruelle-Takens route to chaos (Newhouse et. al., 1978).

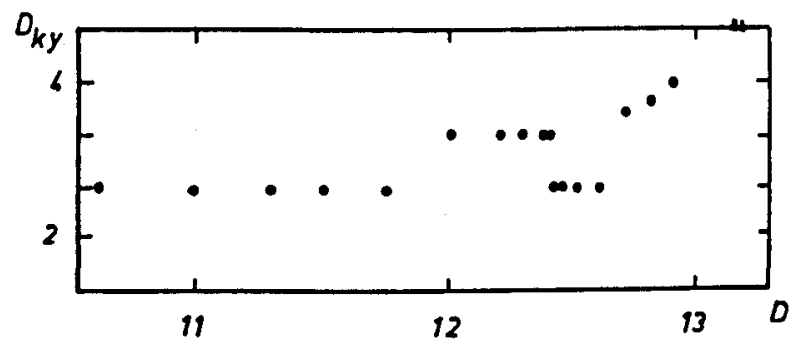

Fig. 4: Kaplan-Yorke dimension $\mathrm{D}_{\mathrm{KY}}$ of (3) in dependence on $\mathrm{D}$ for $q=-0.075$.

The trajectories of the system in the chaotic regime show a complicate amplitude-frequency modulation as well as epoches of extremely low activity (Fig.5). Both features have been observed for solar activity, too.

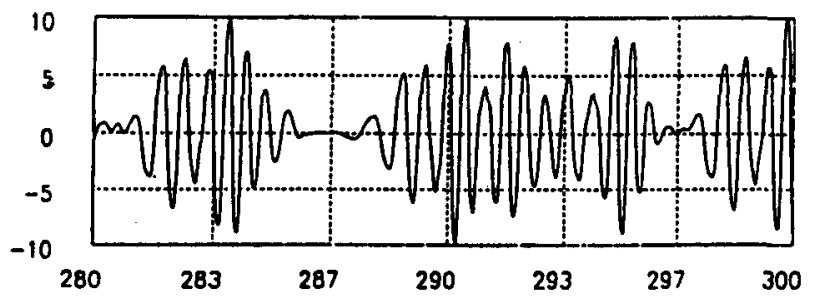

Fig. 5:Component a of a trajectory of (3) in the chaotic regime. 


\section{CONCLUSIONS}

Applying modern techniques of time series analysis we get serious indications that the dynamics of the global solar activity is low-dimensional chaos. Thus, a rather simple nonl inear dynamo model is qualitatively studied exhibiting a $r i c h$ dynamical behaviour from steady state via some bifurcations to a chaotic regime. In the chaotic state this system yields peculiar properties as observed for the solar activity.

Finally, we should like to emphasize that the fitness of this model to describe the global solar activity is not a well-established fact despite the above indications. The investigation of such dynamics is still in its infancy and should be continued by including other stars with activity cycles and other nonl inear dynamo models.

\section{REFERENCES}

1 Eddy, J.A., (1976), Science 286, 1198.

2 Farmer, J.D. and Sidorovich,J.J. (1987), Phys. Rev.Lett. 59,845 .

3 Feudel,U. and Jansen, W. (1988), in: System Analysis and Simulation, Akademie-Verlag, Berlin.

4 Grassberger, D. and Procaccia, I . (1983), Phys.Rev.Lett. 50,346 .

5 Kaneko, K.(1986), Collapse of Tor $i$ and Genesis of Chaos in Dissipative Systems, Korld Scienctific, Singapore.

6 Kaplan, J.L. and Yorke, K. - H, (1979), Comm.Math...Phys. 67,63 .

7 Kurths, J. (1987), Preprint.

8 Kurths, J. and Ruzmaikin, A.A.(1990), Solar Phys. 126,403.

9 Malinetsky, G.G., Ruzmaikin,A.A. and Samarsky, A.A. (1986) Preprint.

10 Newhouse, S., Ruelle,D. and Takens,F. (1978), Comm.Math. Phys. 6435 .

11 Nicolis, G. (1990), Private Communication.

12 Priest, E. (1982), Solar Magnetohydrodynamics, Reidel, Dordrecht.

13 Ruelle,D. (1989),C-haotic Evolution and Strange Attractors, University Press, Cambridge.

14 Shimada, J. and Nagashima, T. (1979), Progr.Theor. Phys.61, 1605 .

15 Takens, F. (1981), in: Lect. Notes in Math.Vol.898, Springer, Berlin.

16 Weiss, N.O. (1988), in: NATO ASI Series C Vol. 236, Kluwer, Dordrecht .

17. Krause, F. and Radler, K. - H: (1980), Mean-Field Magnetohydrodynamics and Dynamo Theory, Akademie-Verlag, Berl in. 